\title{
Entwicklung eines Werkzeugs und des Prozesses für das automatisierte Schaben von Metalloberflächen
}

\author{
Simon Eberle ${ }^{1}$, Kai Oßwald ${ }^{2}$
}

\section{Zusammenfassung}

Das Schaben ist ein Verfahren zur Bearbeitung metallischer Oberflächen und wird bislang ausschließslich manuell angewendet. Der Beitrag handelt von der Entwicklung eines Werkzeugs und des Prozesses für das Schaben von Metalloberflächen. Das im Rahmen eines Forschungsprojekts entwickelte Vorsatzwerkzeug zum Anbau an eine standardisierte CNC-Fräsmaschine bietet unter der Verwendung eines ebenfalls entwickelten Softwaretools die Möglichkeit das Verfahren unabhängig von spezialisierten Fachkräften durchführen zu können. Des Weiteren kann durch die Automation des Verfahrens eine höhere Produktivität realisiert werden. Der Beitrag geht neben dem aktuellen Stand der Technik intensiv auf die Eigenschaften des Vorsatzwerkzeugs, die Entwicklung eines notwendigen spezifischen Softwaretools sowie auf die Qualifizierung des Systems ein. Abschließend werden die gewonnen Ergebnisse in die Thematik eingeordnet und weiteres Forschungspotential ausgewiesen.

\section{Stichwörter}

Schaben, automatisiertes Schaben, Oberflächenfinish, CNC-Technik

\section{Einleitung}

In der Herstellung von Maschinenkomponenten sind neben der Erfüllung von geometrischen Anforderungen auch die Eigenschaften der entsprechenden Werkstückoberfläche relevant. Da diese in der technischen Anwendung häufig entscheidend für deren Funktion sind, werden Oberflächen häufig durch industrielle spanende Finishingverfahren, wie das Schleifen oder das Honen, nachbearbeitet. Darüber hinaus gibt es jedoch weiterhin handwerkliche Verfahren zur Optimierung metallischer Werkstückoberflächen, wie das Schaben, welches bereits seit Jahrhunderten eingesetzt wird. Neben der Erzeugung von ebenen Bauteiloberflächen zeichnet sich das Verfahren durch die besondere Oberflächenstruktur und dadurch entstehende sehr gute tribologische Eigenschaften aus. Des Weiteren generiert das Schaben bei der Erzeugung des Spanabtrags im Vergleich zu anderen abtragenden Verfahren nur einen sehr geringen Wärmeeintrag in die Oberfläche, sodass die Oberflächeneigenschaften des Werkstückes hierdurch nicht nachteilig beeinflusst werden. Diese Vorteile führen unter anderem dazu, dass das Verfahren - trotz der damit verbundenen hohen Personalkosten - bis heute im Bereich von Maschinenführungen und Lagern im Einsatz ist.

1 Simon Eberle, M.Sc., Institut für Werkstoffe und Werkstofftechnologien, Hochschule Pforzheim

2 Prof. Dr. Kai Oßwald, Institut für Werkstoffe und Werkstofftechnologien, Hochschule Pforzheim 


\section{Technologien zur Erzeugung geschabter Oberflächen}

Das Schaben ist nach DIN 8589-9, das „Spanen mit einem Spanwerkzeug (Schaber) zur Veränderung der Werkstückoberfläche, wobei der Schaber entlang dieser Oberfläche geführt und die Spanungsdicke durch die Anpresskraft gesteuert wird.“ Darüber hinaus definiert die zurückgezogene VDI Richtlinie 3220 das Verfahren wie folgt: „Schaben ist das Spanen mit vorzugsweise einschneidigem, nicht ständig im Eingriff stehendem, in einer Hauptrichtung bewegtem Werkzeug zur Verbesserung von Form, Maß, Lage und Oberfläche vorgearbeiteter Werkstücke.“ Infolge der Bearbeitung entstehen unregelmäßig gekreuzte Bearbeitungsspuren, welche eine geschabte Oberfläche auch in Bezug zu den resultierenden Eigenschaften auszeichnen. Die Erzeugung geschabter Oberflächen erfolgt in der Praxis bislang ausschließlich durch handgeführte Systeme.

- Handgeführte Systeme

Die Bearbeitung einer Werkstückoberfläche durch handgeführte Systeme kann hierbei auf zwei unterschiedliche Weisen erfolgen. Dies sind einerseits das ursprüngliche Handschaben und andererseits das Schaben mit Hilfe eines Elektrowerkzeugs. Beim Verfahren des Handschabens wird neben der richtigen Positionierung des Werkzeugs in Ort und Winkellage die Vorschub- und Schnittbewegung durch körperliche Betätigung des Werkers durchgeführt. Infolgedessen wird sowohl die Schnittkraft als auch die Passivkraft durch den Werker aufgebracht, welche zu einer hohen körperlichen Anstrengung führen. Unsere Arbeitsgruppe befasste sich bereits in der Vergangenheit mit den Besonderheiten des Verfahrens und führte Forschungen hinsichtlich der auftretenden Prozessparameter [4], der angewendeten Schabstrategien und der resultierenden Oberflächeneigenschaften [5] durch.

Eine Alternative zu der körperlich anstrengenden Methode des Handschabens stellt das „maschinelle“ Schaben mittels Elektrowerkzeug dar. Hierbei erzeugt ein Elektromotor über ein Getriebe eine oszillierende translatorische Bewegung, welche der Schnittbewegung entspricht. Auf Basis dieser Grundbewegung führt nun der Werker die Vorschubbewegung bzw. die Positionierung in Ort und Lage weiterhin durch. Die Nutzung des Elektrowerkzeuges ermöglicht eine höhere Produktivität und wird zumeist für die Schruppbearbeitung großflächiger Bauteile eingesetzt. Darüber hinaus kann das Elektrowerkzeug bei geeigneter Anwendung auch für Schlichtoperationen eingesetzt werden.

Da die Anwendung des Verfahrens jedoch speziell qualifizierte Fachkräfte erfordert, hat seine Anwendung aufgrund der hohen Personalkosten des Gewerks und des Mangels an entsprechend ausgebildeten Mitarbeitern trotz der Verfahrensvorteile in den letzten Jahrzehnten stark abgenommen. Infolgedessen haben sich bereits unterschiedliche Forschergruppen damit beschäftigt, die Technologie des Schabens auf Basis unterschiedlicher Automatisierungsansätze zu realisieren.

\section{- Automatisierungsansätze}

Bisherige Konzepte zur Automatisierung des Schabprozesses eint, dass sie auf eigenständigen, autarken Maschinen beruhen. Tong et al. [6] stellen ein System in Anlehnung an eine Werkzeugmaschine vor. Der Aufbau verfügt über drei translatorische Achsen zur Positionierung im Raum und eine Drehachse zur Ausrichtung des Werkstücks in der X-/Y- Ebene. Die Schabbewegung wird hierbei durch einen Pneumatikzylinder realisiert. Ein weiteres System wurde von Tsutsumi et al. [7] entwickelt. Hierbei wird ein federvorgespannter Schabmeißel über einseitig gelagerte, auskragende Maschinenachsen bewegt. Infolgedessen müssen bei dieser Bauweise die Achsen zur Positionierung auch die Schnitt- und Passivkraft für den Schabprozess aufbringen. Der großflächige Maschinentisch dieses Prototyps ermöglicht die Befestigung und Bearbeitung großer Bauteile. Um die Bearbeitung von großflächigen Bauteilen zu erleichtern und die Bindung an die Einschränkungen durch die Maschine zu reduzieren, entwickelten Lin et al. [8] ein mobiles System in Gantrybauweise bzw. Tsai et al. [9] ein System basierend auf einem starren Portal. Die von Lin et al. [8] entwickelte Methode ermöglicht eine höhere Flexibilität, da der Aufbau mit einem geringen Eigengewicht von rund $30 \mathrm{~kg}$ um das zu bearbeitende Werkstück platziert werden kann. Alternativ hierzu vereinigt die Portalbauweise von Tsai et al. [9] die gesamte Bewegungseinheit kompakt in einer Baugruppe. Dies führt dazu, dass große 
Bauteile mit einem eingeschränkten Bearbeitungsbereich bearbeitet werden können. Darüber hinaus haben sich die bereits erwähnten Forschergruppen auch mit der Erfassung der Oberfläche mittels CCD (Charge-Coupled Device)-Zeilenkamera oder Lasertriangulation und der Implementierung der daraus gewonnenen Erkenntnisse in den jeweiligen Maschinencode befasst (bspw. in [10]). Diese Maßnnahme ermöglicht die Bereiche, welcher eine Überarbeitung bedürfen zu identifizieren und gezielt zu bearbeiten. Dennoch liegen derzeit keine Informationen über ein kommerziell verfügbares automatisiertes System zur Herstellung geschabter Oberflächen vor.

\section{Entwicklung eines Vorsatzwerkzeuges für eine CNC- Fräsmaschine}

Um einen reproduzierbaren und produktiven Schabprozess zu ermöglichen, entwickelt die Hochschule Pforzheim zusammen mit dem Kooperationspartner ROMAI Robert Maier GmbH, Vaihingen/Enz-Horrheim eine Methode mit der Intention das Verfahren zu modernisieren und an die aktuellen Randbedingungen der Wirtschaftlichkeit und des bestehenden Personalangebotes anzupassen. Im Fokus steht daher die Entwicklung einer automatisierten Lösung zur Bearbeitung der Bauteile, sodass das Verfahren in einem weiten Einsatzgebiet unabhängig von speziell geschultem Personal Anwendung finden kann. Damit das neue System einfach in bestehende Fertigungsabläufe integriert werden kann, erfolgte nach Abwägung verschiedener Lösungsvarianten die Entscheidung die Automatisierung mittels eines Vorsatzwerkzeuges auf bestehende Maschinen umzusetzen. Die Basis des automatisierten Prozesses stellt hierbei eine handelsübliche drei- oder fünfachsige CNC-Fräsmaschine mit einem von ROMAI entwickelten und gefertigten Vorsatzwerkzeug dar. In der Folge erprobt die Hochschule Pforzheim das Werkzeug und entwickelt einen entsprechenden Fertigungsprozess.

Das Werkzeug, welches in Bild 1 schematisch dargestellt ist, wird an die Spindeleinheit der Fräsmaschine angebaut und mittels einer Standardschnittstelle angetrieben. Das im Werkzeug verbaute Spezialgetriebe wandelt hierbei die eingeleitete senkrechte Rotationsbewegung über verschiedene Zwischenstufen in eine oszillierende translatorische Bewegung um. Der Bewegungsablauf kann der Detailansicht in Bild 1 entnommen werden.

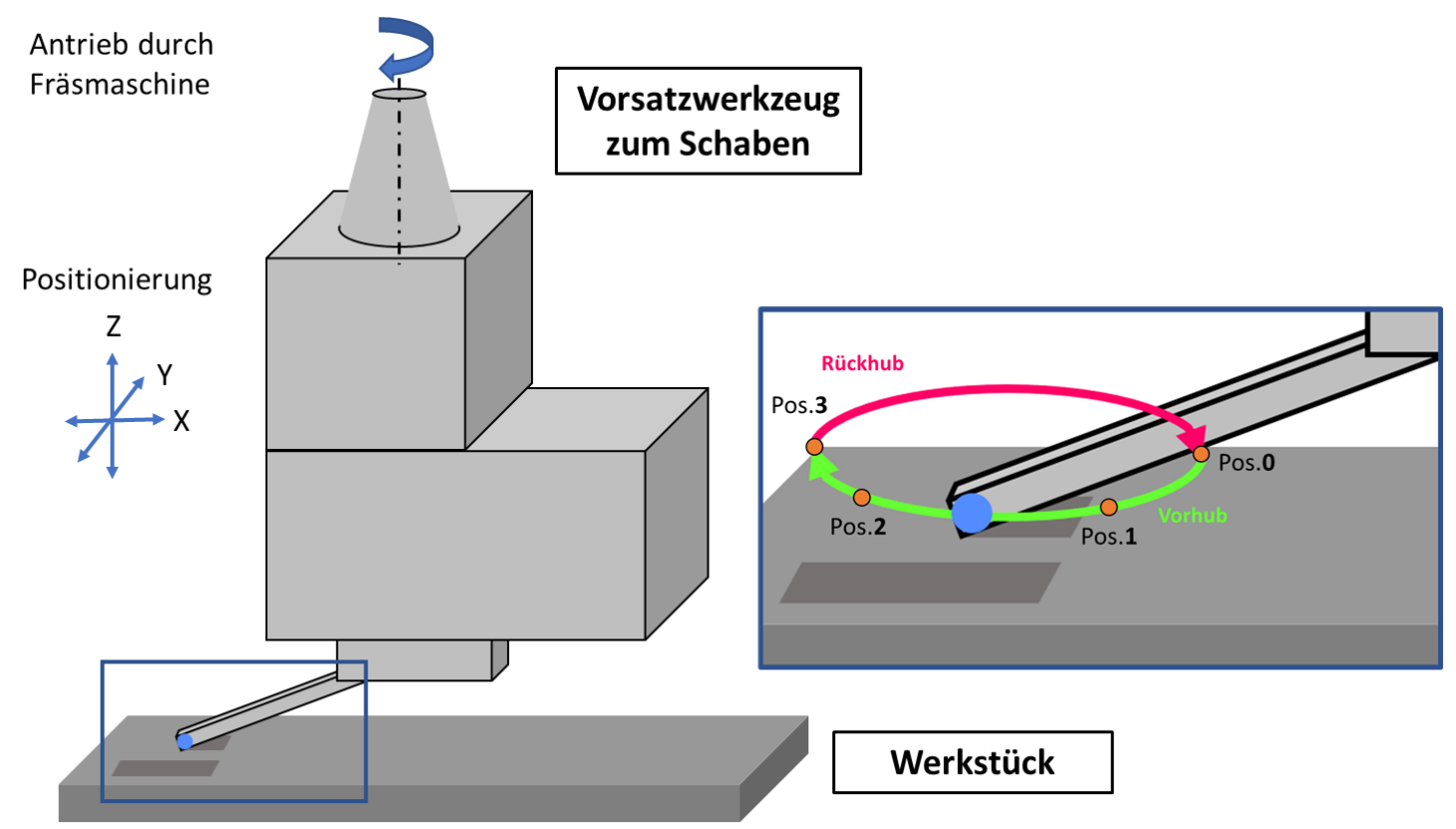

Bild 1: Schematische Darstellung des Schabwerkzeugs 
Eine Besonderheit ist, dass die Schneide des Meißels abweichend von den bisher bekannten Schabverfahren eine elliptische Bewegungskurve verfolgt und daher aufgrund des integrierten Abhebehubes in Z-Richtung den Rückhub ohne eine Werkstückberührung vornimmt. Somit entsteht ein Bewegungsablauf des Schabmeißels, welcher vier wesentliche Zwischenpositionen durchläuft. Diese Positionen sind in auch in der Detailansicht der schematischen Abbildung dargestellt. Der immer wiederholende Bewegungsablauf startet mit dem Beginn des Vorhubs in der Pos.0. In Abhängigkeit der gewählten Parameter erfolgt darauf hin bei Pos.1 der Eintritt des Meißels in den Werkstoff und bei Pos. 2 der Austritt aus dem Werkstoff. In Folge ist die Erstellung der einzelnen Schabfurche beendet und der Schabmeißel startet in Pos.3 den Rückhub, um wieder zum Ausgangspunkt des Zyklus zu gelangen. Die Ausprägung der Bewegungskurve kann durch eine Modifikation im Werkzeug verändert werden. Darüber hinaus kann die Länge des Schabhubes sowie die Winkelorientierung des Werkzeuges gegenüber dem Werkstück am Werkzeug selbst eingestellt werden. Im Rahmen der Schabbearbeitung wird das Werkzeug mittels der drei Koordinatenachsen der Fräsmaschine in X-, Y- und Z- Richtung positioniert, wodurch die Bearbeitungsstrategie der zu bearbeitenden Fläche auf Grundlage eines zuvor übermittelten NC- Programmes abgearbeitet wird.

\section{Entwicklung eines Softwaretools zur automatisierten Erzeugung von NC-Programmen}

Die Entwicklung des Vorsatzwerkzeugs stellt in Bezug zu einer Komplettlösung für das automatisierte Schaben nur einen Bestandteil dar. Daher wurde für die Automatisierung des Prozesses ein Softwaretool entwickelt, sodass der Anwender eine einfache Umsetzung des automatisierten Schabens ohne spezielle Vorkenntnisse vornehmen kann. Das Tool kann auf Basis der im Interface eingegebenen Daten ein an die Bearbeitungsfläche angepasstes NC- Programm im „G-Code“ nach DIN 66025/ISO 6983 erstellen. Diese Aufgabe übernimmt in konventionellen Prozessen normalerweise eine CAM-Software (Computer Aided Manufacturing). Da das Schaben (bspw. im Vergleich zum Fräsen) eine ganze Reihe von Besonderheiten aufweist, werden diese im eigens erstellten Softwaretool berücksichtigt und entsprechend umgesetzt.

\subsection{Programmstruktur}

Das Softwaretool (mit dem internen Namen „G-Code Generator") ist eine WPF (Windows Presentation Foundation)-Anwendung, welche in der Softwareumgebung von Visual Studio, Microsoft entwickelt wurde. Hierbei ist für das User Interface die Programmiersprache XAML (Extensible Application Markup Language) und für das Backend die Programmiersprache VBA (Visual Basic for Application) verwendet worden. Die Programmstruktur des Softwaretools, welche auch in Bild 2 dargestellt ist, beginnt mit dem Öffnen der Anwendung in der Windowsumgebung. Ein User- Interface wird geöffnet und der Anwender kann seine Daten für das automatisierte Schaben eingeben. Hierbei sind die eingegeben Daten in zwei Kategorien gegliedert: in die zwingend erforderlichen und die optionalen Daten. $\mathrm{Zu}$ den zwingend erforderlichen Daten gehören beispielweise Daten zum Werkstück, wie die Maße der Bearbeitungsfläche, die Schabtiefe und die Schabhubdichte sowie Werkzeug- und Maschinendaten, wie der Richtungswinkel, die Schabhublänge und die Hubfrequenz. Darüber hinaus können die optionalen Daten in einem separaten Fenster eingegeben werden und beinhalten Daten, welche vom Standard abweichen. Hierzu zählen beispielsweise Inhalte zu den Maschinengrenzwerten, zu der eingesetzten Maschine, zu den Nullpunkten oder zur Wahl der Bearbeitungsrichtung. Nach Abschluss der Eingabe werden die Parameter nach den festgesetzten Anforderungen geprüft und die Vorschubgeschwindigkeit berechnet. Im Falle von Unstimmigkeiten fordert eine Warnmeldung den Anwender zur Korrektur der Daten auf. Sofern bei der Berechnung der Vorschubgeschwindigkeit keine Maschinengrenzwerte überschritten worden sind, kann nun der Button „Code generieren“ aktiviert und die 
Berechnung des G-Code angefordert werden. Da die Berechnung in Abhängigkeit verschiedener Auswahlmöglichkeiten erfolgt, führt das Programm verschiedene Abfragen durch. Die hierfür notwendigen Abfragen sind in Bild 2 mittig in hellblau dargestellt und beziehen sich auf zuvor festgelegte Auswahlen. Diese betreffen Aspekte zur Maschinensteuerung, zu einer möglichen Nullpunktverschiebung, zur Bauart der Fräsmaschine (3- oder 5-achsig) und zur Festlegung des Werkzeugnullpunkts sowie zur gewählten Bearbeitungsrichtung (Gleichlauf/Gegenlauf). Nach abgeschlossener Berechnung wird nun der Code zur Durchsicht angezeigt und kann nach Festlegung des Dateipfads per Click exportiert werden. Abschließend kann die Export-Datei weiter an die Steuerung der Fräsmaschine übermittelt und die Bearbeitung initiiert werden.
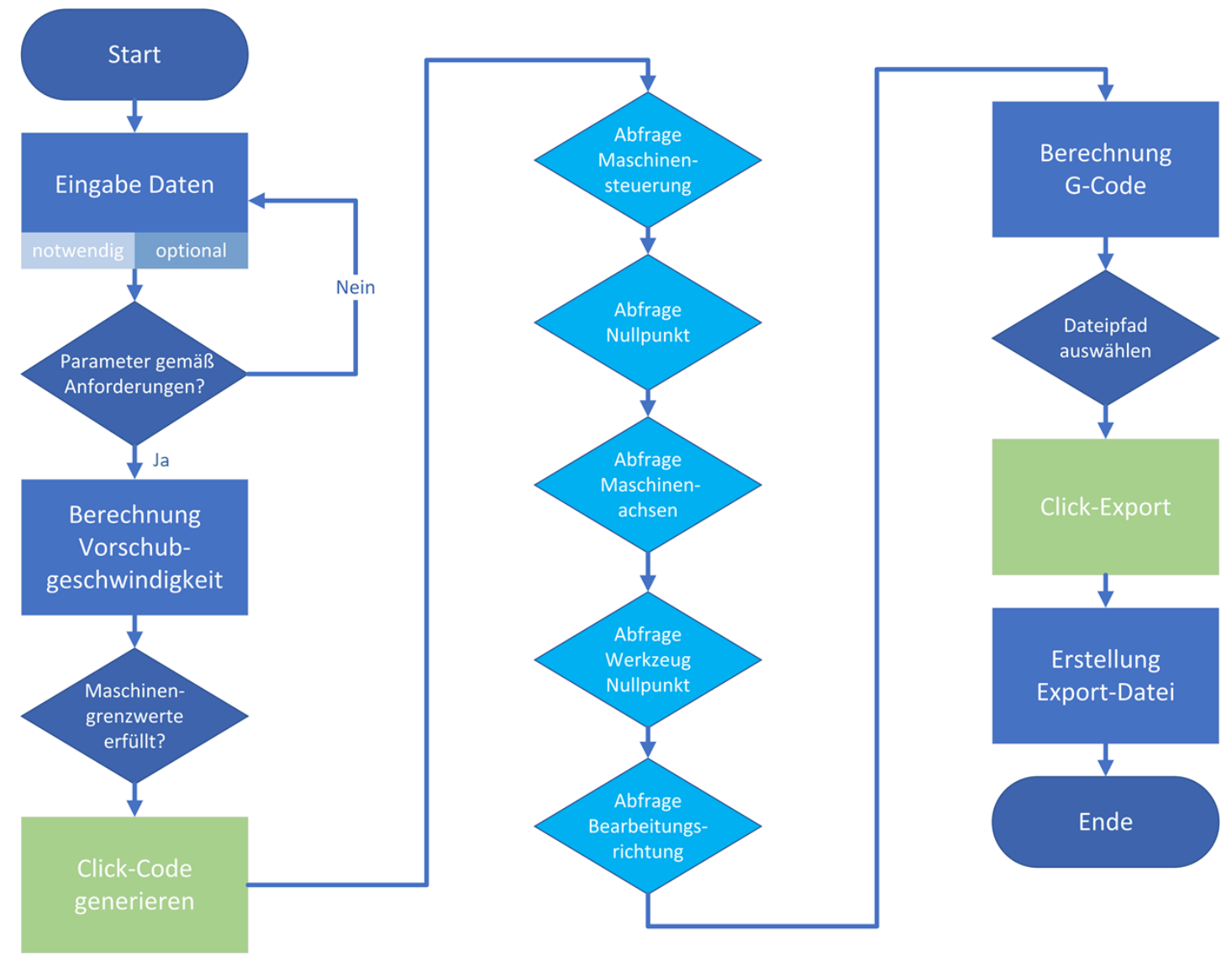

Bild 2: Programmstruktur Softwaretool

\subsection{Software Kompensationen}

Im Rahmen der G-Code-Berechnung führt die Software zur Berücksichtigung der schabspezifischen Besonderheiten verschiedene Kompensationen durch. Sie sind in zwei Kategorien gegliedert. Dies sind einerseits die Kompensationen mit dem Bezug zum Werkzeug und andererseits die Ausgleichsmaßnahmen, welche in Verbindung mit der Bewegung stehen. Die werkzeugspezifischen Aspekte betreffen hierbei sowohl die Positionierung in der X-/Y-Ebene, als auch die Zustellung in Z-Richtung. In Abhängigkeit der verwendeten Meißellänge und des gewählten Richtungswinkels des Werkzeugs muss die Abstandsänderung in X- und Y-Richtung zwischen der Meißelspitze und der Spindelmitte des Werkzeugs, welche den Referenzpunkt bildet, zur richtigen Positionierung des Werkzeugs berücksichtigt werden. Da darüber hinaus das Werkzeug im Bearbeitungsprozess eine Nachgiebigkeit aufweist, muss diese in der Zustellung in Z-Richtung durch eine entsprechende Federkonstante ebenfalls berücksichtigt werden. Des Weiteren sind durch die systembedingten Bewegungsabläufe weitere Maßnahmen notwendig. Durch die Überlagerung der Schabhubgeschwindigkeit $v_{t}$ mit der 
Verfahrgeschwindigkeit $v_{f}$ des Werkzeugs entstehen Auswirkungen auf die Länge und den Richtungswinkel der Schabfurche, welche in Bild 3 dargestellt sind. Im Fall einer positiven Verfahrgeschwindigkeit erfolgt die Bearbeitung im Gleichlauf. Hierdurch ergibt sich ein größerer effektiverer Richtungswinkel $\rho$ sowie eine erhöhte Länge der Schabfurche (rote Schraffierung). Bei umgekehrter Verfahrrichtung des Werkzeugs (Gegenlauf) entsteht ein geringerer effektiver Richtungswinkel $\rho$ sowie eine geringere Länge der Schabfurche (orange Schraffierung). Um diesen Effekt zu eliminieren muss die Software in Abhängigkeit des Verhältnisses der Drehzahl zu der Verfahrgeschwindigkeit den Einstellrichtungswinkel $\varphi$ berechnen und in den Code implementieren.

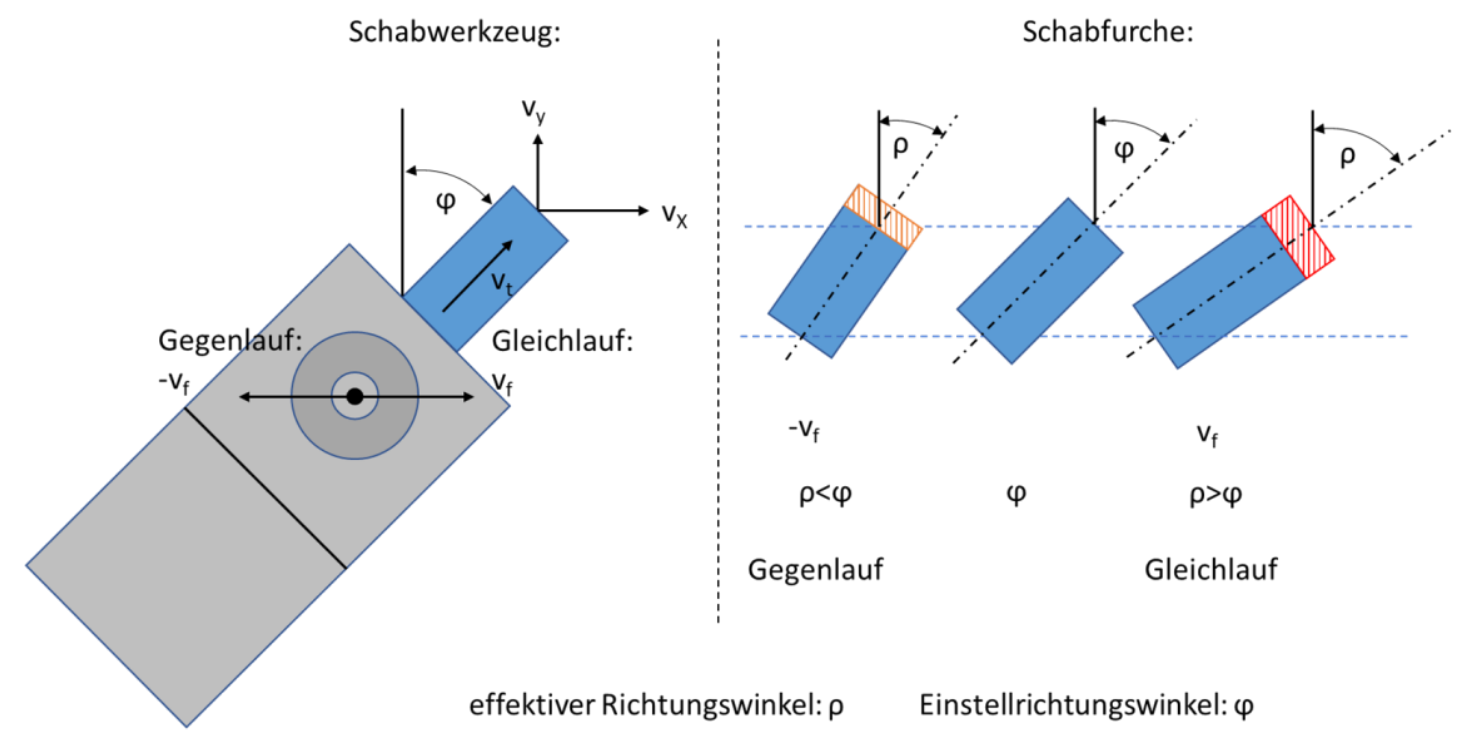

Bild 3: Kompensationen im Kontext zur Bewegung

\subsection{Beispielprogramm}

Im Folgenden wird exemplarisch der "G-Code" für die Ansteuerung einer CNC-Fräsmaschine nach DIN 66025/ISO 6983 zum automatisierten Schaben vorgestellt. Hierbei umfasst der Code, welcher auszugweise in Bild 4 dargestellt ist, eine flächige Überschabung des Bauteils mit einem Durchgang unter dem Winkel von $+45^{\circ}$ und einem weiteren Durchgang in einem Winkel von $-45^{\circ}$. Der Rahmen eines entsprechenden NC- Programmes wird durch die Anfangs- und Schlusssätze, welche in grau eingefärbt sind, verkörpert. Hierbei wird zu Beginn ein entsprechendes Programm benannt und grundlegende Definitionen zu den Maßeinheiten, zur Wahl des Koordinatensystems oder zur Aufhebung von bestehenden Kompensationen getroffen. Im darauffolgenden Teil (grüne Kennzeichnung) werden dem Bediener nochmals die wesentlichen Prozessparameter, wie zum Beispiel Informationen über die beabsichtigte Hubfrequenz, die Vorschubgeschwindigkeit oder die tatsächliche Schabhubdichte in Form von Kommentaren mitgeteilt. Da dieses Beispielprogramm für die Nutzung an einer 3-Achs-Maschine vorgesehen ist, muss der Bediener am programmierten Halt (rote Kennzeichnung) den Winkel des ersten Durchgangs der Überschabung händisch am Werkzeug einstellen. Nach Abschluss dieser Maßnahme kann das Programm fortgesetzt und die Maschinenspindel mit der gewünschten Drehzahl und dem gewünschten Vorschub im Rechtslauf (gelbe Kennzeichnung) angewählt werden. Im Folgenden wird die Fläche des Bauteils nun reihenweise bearbeitet. Hierbei wird der Schabmeißel an die jeweilige Startposition der Reihe in der X-/Y- Ebene positioniert, die Zustellung in Z-Richtung vorgenommen, die Schabung der Reihe durchgeführt und abschließend der Meißel wieder von der Werkstückoberfläche abgehoben. Dieser Vorgang (blaue Kennzeichnung) wird nun bis zur vollständigen Bearbeitung des Bauteils wiederholt. Nach Abschluss des ersten Durchgangs wird das Werkzeug nun zu einem Werkzeugwechselpunkt gefahren (violette Kennzeichnung) und ein programmierter Halt (rote 
Kennzeichnung) vorgenommen. Nun kann der Bediener ähnlich wie zu Beginn des Programmes den Richtungswinkel am Werkzeug für den zweiten Durchgang einstellen. Im Falle, dass eine 5-Achs-Maschine für die Bearbeitung verwendet wird, kann auf die programmierte Halte verzichtet und stattdessen die Winkeländerung mit Hilfe der Maschinen C-Achse durchgeführt werden. Im weiteren Verlauf kann nun der zweite Teil der Überschabung vergleichbar zum ersten Teil mit abschließender Positionierung zum Werkzeugwechselpunkt und Beendigung des Programmes vorgenommen werden.

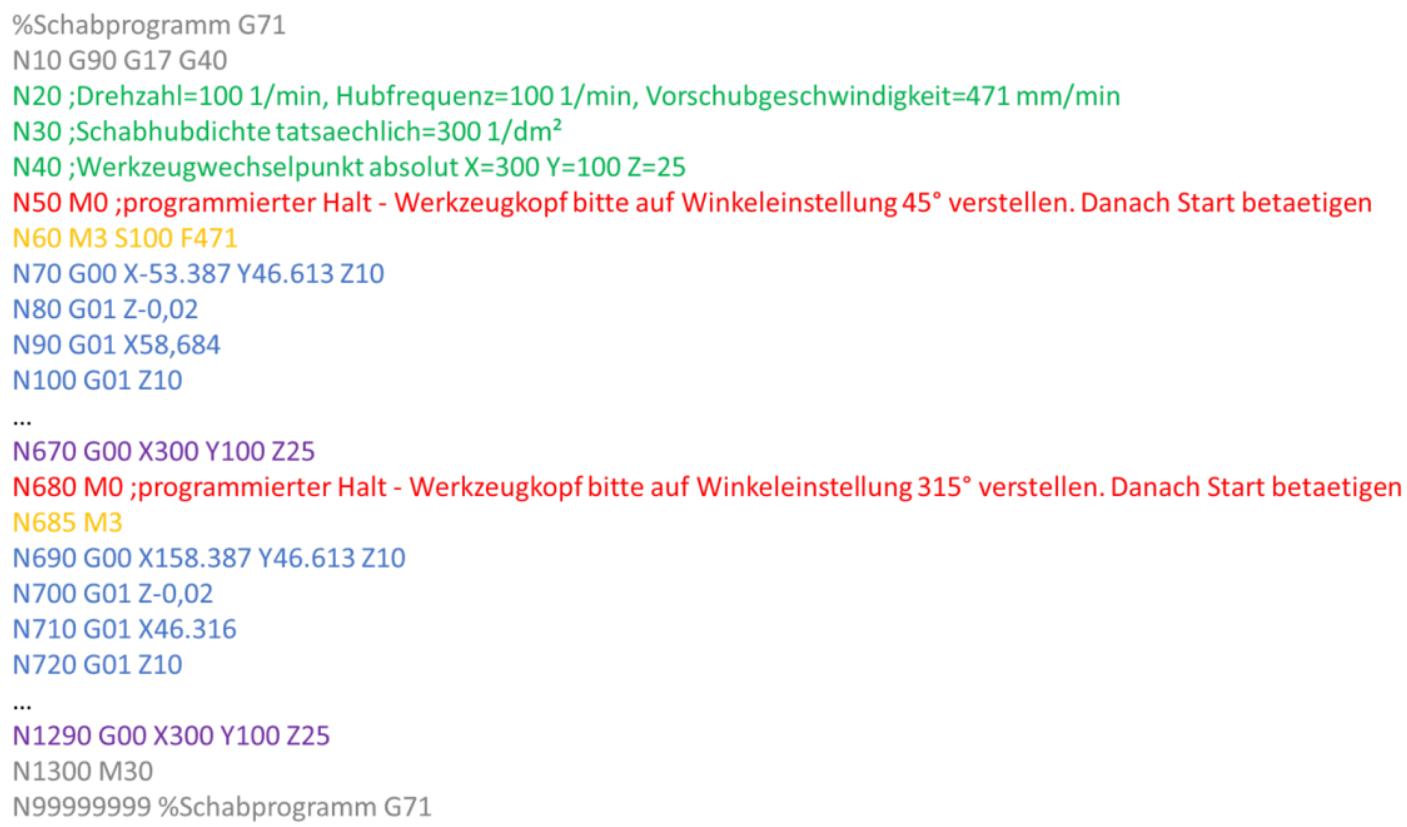

Bild 4: Beispielprogramm

\section{Qualifizierung des Systemverhaltens}

Um abschließend die Einsatzfähigkeit und die Eignung des automatisierten Systems für die industrielle Schabbearbeitung nachzuweisen, werden mittels eines Prüfstandes verschiedene Kenngrößen erfasst. Diese umfassen die auftretenden Kräfte, die Bearbeitungstiefen, die Bewegungsabläufe und die erzeugten Oberflächen, welche analysiert, ausgewertet und mit den Daten des bisher bekannten Handschabens bzw. Elektroschabens verglichen werden. Hierzu ist geeignete Messtechnik notwendig, welche im Folgenden näher vorgestellt wird. Auf dieser Basis können der automatisierte Prozess sowie der Prototyp des Schabwerkzeugs in Zusammenarbeit mit dem Industriepartner intensiv untersucht, kontinuierlich optimiert und mögliche Anwendungsbereiche ermittelt werden.

\subsection{Messtechnik}

Die Messtechnik zur Untersuchung der unterschiedlichen Prozessparameter besteht aus verschiedenen Sensoren sowie Hardware und Software zur Erfassung der Daten. Zum Einsatz kommen hierbei zwei taktile Wegmesssensoren, ein Drehwinkelsensor, eine Kraftmessdose sowie ein Schaltkontakt. Die Sensoren sind mittels 3-D gedruckter Vorrichtungen an das Werkzeug selbst oder auf den Maschinentisch der CNC- Fräsmaschine appliziert. Eine Übersicht über die Messtechnik ist dem Bild 5 zu entnehmen. Hierbei ermöglichen die zwei taktilen Messtaster des Herstellers Keyence (Typ GT2- H12 / GT2-H32) eine Erfassung des Abhebehubs in Z- Richtung bzw. die Erfassung der Schabhublänge, sodass der reale Bewegungsablauf des Schabmeißels erfasst werden kann. Des Weiteren wird mit einem 
Positionssensor des Herstellers Honeywell (Typ RTY360HVEAX) die Position des Drehwinkels in Bezug zum Schabhub erfasst. Hierzu ist die Antriebswelle des Sensors formschlüssig mit einer Zwischenwelle des Werkzeugs verbunden. Um darüber hinaus eine Aussage über die Berührung des Schabmeißels mit der Werkstückoberfläche treffen zu können, ist in den Messaufbau ein Kontaktstromkreis mit Kontroll-LED eingerichtet worden, welcher bei Berührung des Schabmeißels auf der Werkstückoberfläche geschlossen wird. Infolge kann über den Spannungsabfall die entsprechende Kontaktierung ausgewertet werden. Abschließend werden noch die entstehenden Prozesskräfte mittels einer Kraftmessplattform in allen drei Raumrichtungen erfasst. Der hierfür verwendete Sensor, ist ein Dynamometer des Herstellers ME-Systeme (Typ K3D120) und besitzt einen Messbereich von $\pm 500 \mathrm{~N}$. Die zu erfassenden Messdaten werden auf Basis eines LabVIEW-Programmes und der dazu notwendigen Hardware des Herstellers NI erfasst und in entsprechende Datenfiles zur weiteren Auswertung abgespeichert.

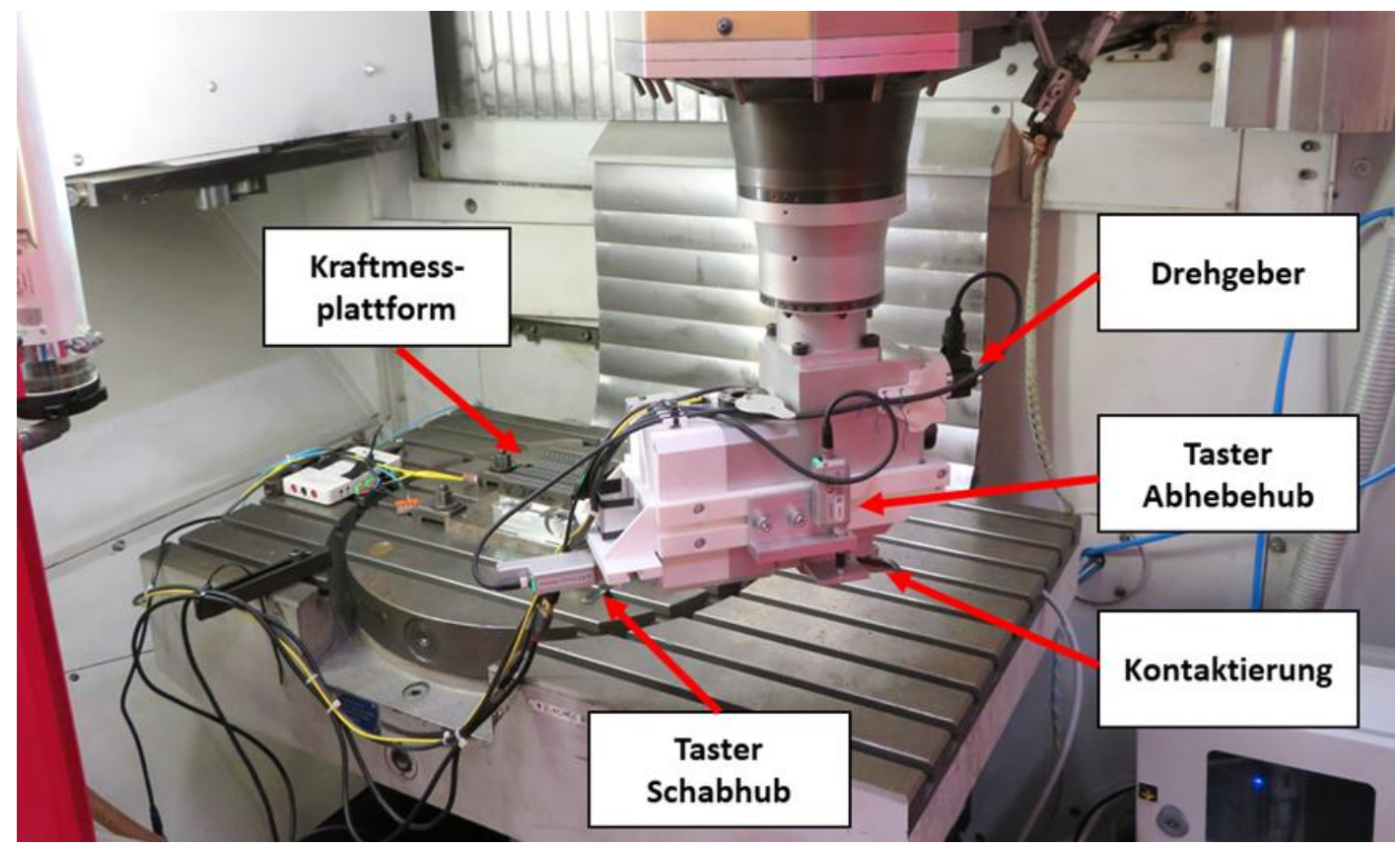

Bild 5: Messtechnik zur Systemqualifizierung

\subsection{Analyse der Versuchsreihen}

Im Rahmen der durchgeführten Versuchsreihen können verschiedene Prozessparameter, wie zum Beispiel die Drehzahl, der Vorschub, die Zustelltiefe oder die Bewegungsart variiert und deren Auswirkungen ermittelt werden. Somit kann durch die bereits vorgestellte Messtechnik eine detaillierte Betrachtung zwischen den Prozesseingangs- und Ausgangsgrößen erfolgen und Zusammenhänge bzw. Abhängigkeiten zwischen den eingestellten Größen und den resultierenden Größen können festgestellt werden. Im Folgenden werden nun exemplarisch verschiedene Ergebnisse der Versuchsreihen vorgestellt und deren Erkenntnisse erläutert.

Das Bild 6 zeigt den realen Bewegungsablauf des Schabmeißels in der aktuellen Konfiguration des Werkzeugs im Bewegungsmodus „Gegenlauf“. Hierbei werden die erfassten Messdaten der Schabhublänge auf der X-Achse und des Abhebehubs in Z-Richtung auf der Y-Achse über mehrere Zyklen hinweg in Form eines X-/Y- Diagrammes aufgetragen und qualitativ dargestellt. Dabei ist zuerkennen, dass die Bewegungskurve von einer elliptischen Bahn abweicht und stattdessen im Bereich des Vorhubs eine eher geradlinige aufsteigende Bewegung aufweist. Darüber hinaus sind auch deutliche Unterschiede im Verlauf zwischen dem Bewegungsablauf mit Werkstückkontakt (blaue Kurven) und dem Bewegungsablauf ohne Werkstückkontakt (orange Kurven) festzustellen. Im Falle einer 
Materialbearbeitung steigt der Verlauf des Abhebehubs mit Beginn des Vorhubs kontinuierlich an und nähert sich erst nach dem Materialaustritt wieder der unbelasteten Bewegungskurve an. Infolge ist die Einfederung des Systems bei der Wahl der Zustelltiefe zu beachten.

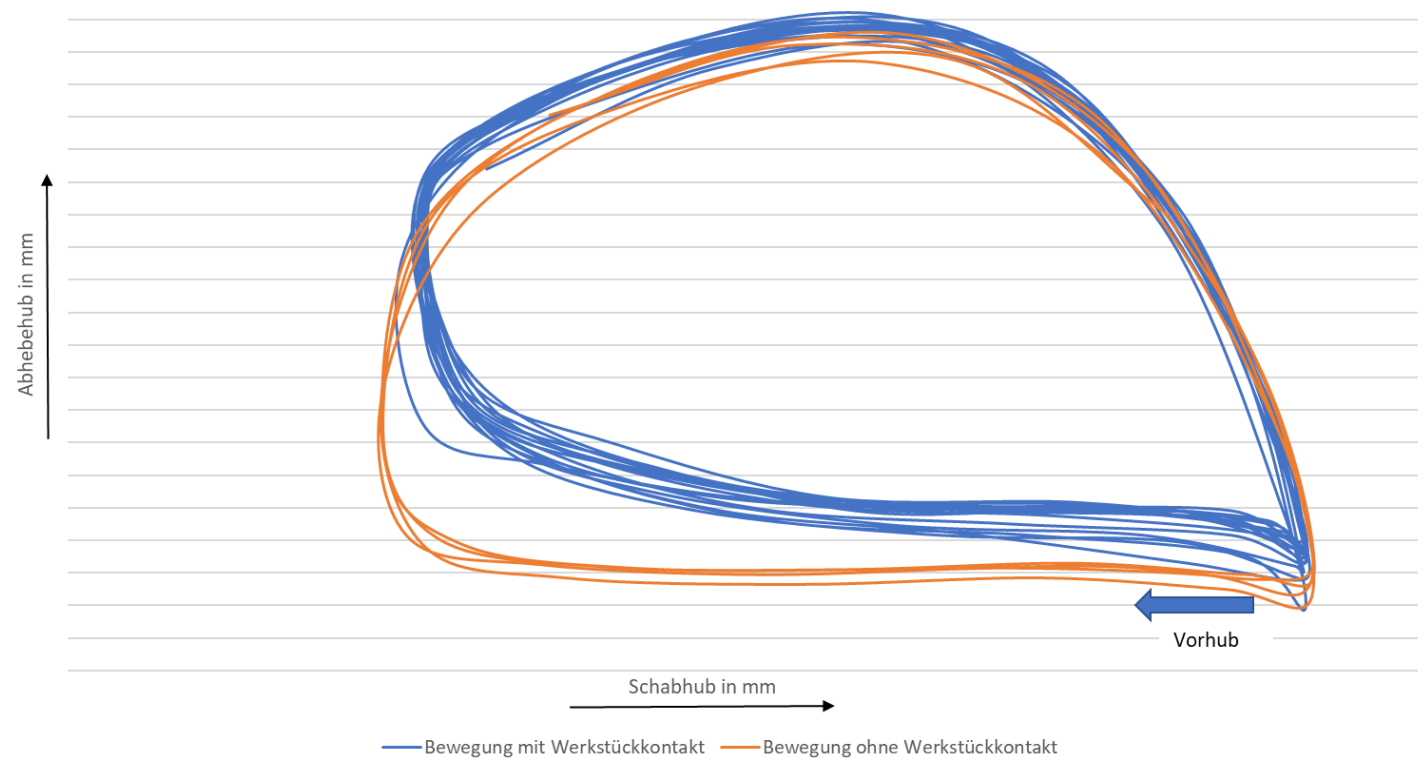

Bild 6: Bewegungsablauf Schabmeißel

Über den Bewegungsablauf hinaus ist auch eine Betrachtung der Prozesskräfte von hoher Bedeutung. Das nachfolgende Bild 7 stellt die resultierende Schnittkraft $F_{c}$ (orange) und die Passivkraft $F_{p}$ (blau) über der Zeit für eine Schabreihe dar. Hierbei können dem Diagramm die Kräfte jedes einzelnen Schabhubs entnommen werden. Die Daten entstammen einer Versuchsreihe, bei welcher die Messdaten bei einem Hub von $15 \mathrm{~mm}$ und einer Zustelltiefe von 0,04 mm erfasst wurden. Die Abweichungen in der Schnittkraft sowie in der Passivkraft gegen Ende der Schabreihe können durch einen geringeren Materialabtrag aufgrund von Unebenheiten des Werkstücks erklärt werden. Die Auswertung zeigt somit eine plausible Größenordnung der Schnitt- und Passivkräfte für eine Schruppbearbeitung.

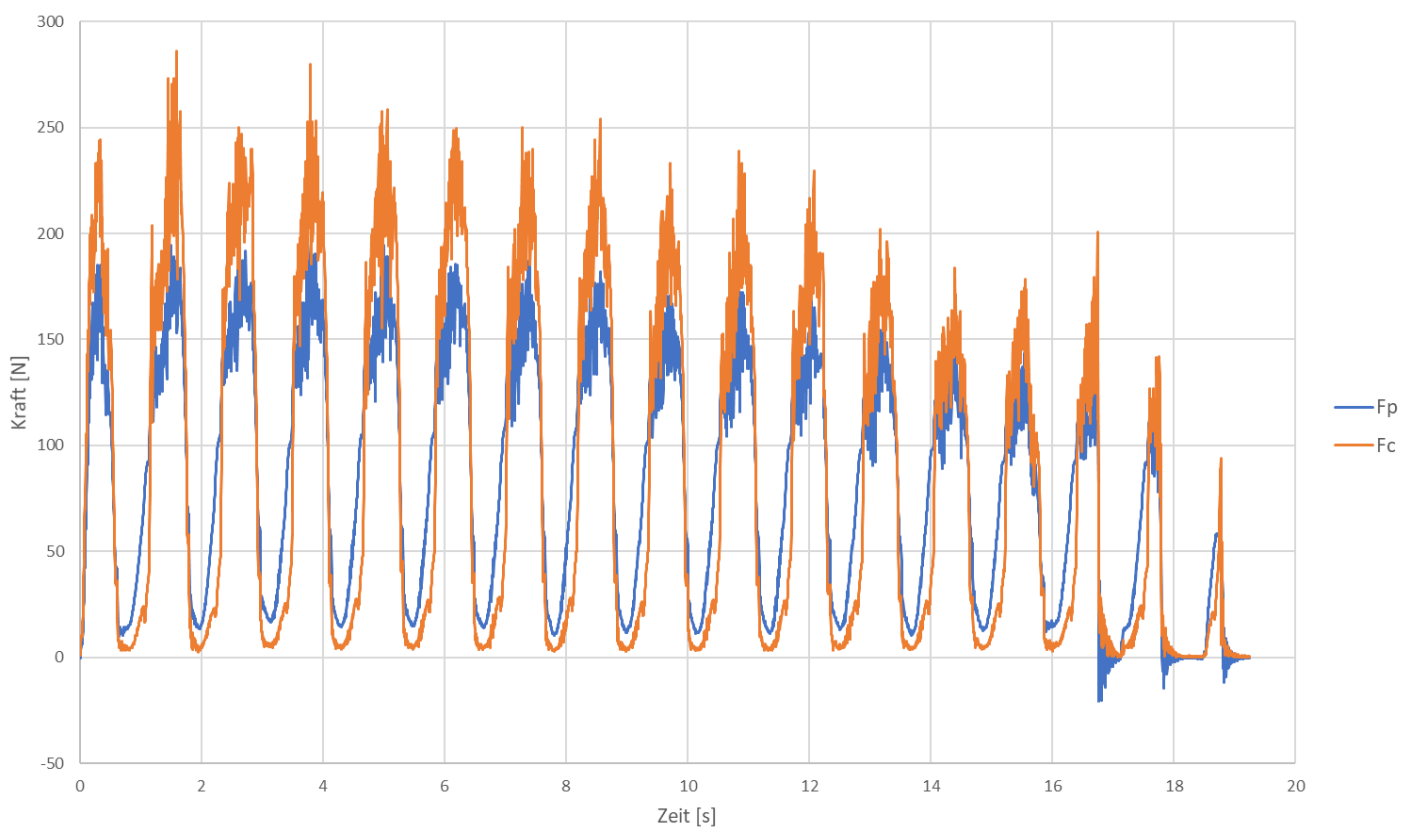

Bild 7: Kraftverlauf einer Schabreihe 


\section{Fazit und Ausblick}

Unsere Forschungen zum automatisierten Schaben haben gezeigt, dass das bislang handwerkliche Fertigungsverfahren des Schabens prinzipiell automatisierbar ist. Das aktuelle System ist bereits in der Lage, erfolgreich Bauteile zu bearbeiten und somit geschabte Strukturen auf Werkstücke aufzubringen. Hierbei liegt derzeit der aktuelle Schwerpunkt primär auf der Schruppbearbeitung. Da die Schabbearbeitung durch einen Facharbeiter einen iterativen Prozess darstellt, nähert dieser sich schrittweise an die gewünschten Anforderungen hinsichtlich der Ebenheit und Lage. Diesen Prozess bilden wir derzeit noch nicht ab und sind infolgedessen auf die Genauigkeit unserer Basismaschine angewiesen. Dennoch stellt die Automatisierung auf Basis einer CNC-Fräsmaschine eine geeignete Lösung dar. Somit kann der Prozess des Schabens ohne große Investitionen mit Hilfe des entwickelten Vorsatzwerkzeugs und des Softwaretools anwenderfreundlich in bestehende Fertigungsabläufe integriert werden.

Um das System weiter zu entwickeln, sollte sich zukünftige Forschungsarbeit auf die Erzeugung geschabter Flächen auf Ebenheit und Lage fokussieren. Darüber hinaus weist auch die Untersuchung der tribologischen Eigenschaften von geschabten Oberflächen im Vergleich zu anderen Finishingverfahren weiteres Forschungspotenzial auf. Infolgedessen können die Verfahrensvorteile des Schabens detaillierter evaluiert und in der Praxis gezielter zum Einsatz gebracht werden.

\section{Literatur}

[1] DIN 8589-9:2003-09, Fertigungsverfahren Spanen -

Teil 9: Schaben, Meißeln - Einordnung, Unterteilung, Begriffe

[2] VDI 3220:1960-03, Gliederung und Begriffsbestimmungen der Fertigungsverfahren, insbesondere für die Feinbearbeitung

[3] Schmid,W. Schaben. in Günter Spur - Handbuch Spanen (2014); Carl Hanser Verlag GmbH \& Co. KG: München, Germany, 2014

[4] Oßwald, K., Lochmahr, I., Bagci, Y., \& Saile, P. (2018). Investigation into Hand Scraping: A Microanalysis. Journal of Manufacturing and Materials Processing, 2(4), 76.

[5] Oßwald, K., Gissel, J. C., \& Lochmahr, I. (2020). Macroanalysis of Hand Scraping. Journal of Manufacturing and Materials Processing, 4(3), 90.

[6] Tong, H. F., Liu, W., Chi, Y., \& Wang, W. (2014). Design of automatic scraping system. In Advanced Materials Research (Vol. 853, pp. 625-630). Trans Tech Publications.

[7] Tsutsumi, H., Yamada, R., Kyusojin, A., \& Nakamura, T. (2007). Development of an Automatic Scraping Machine with Recognition for Bearing of Scraped Surfaces - Construction of Automatic Scraping Machine -. In Towards Synthesis of Micro-/Nano-systems (pp. 355-356). Springer, London.

[8] Lin, Y., Yeh, C. Y., Shiu, S. C., Lan, P. S. \& Lin, S. C. (2018). The design and feasibility test of a mobile semi-auto scraping system. The International Journal of Advanced Manufacturing Technology. Springer, London.

[9] Tsai, Y. C., Li, J. H., Lee, J. N., \& Jywe, W. Y. (2012). Development and implementation of automatic scraping mechanism. Advanced Science Letters, 8(1), 211-215.

[10] Hsieh, T. H., Jywe, W. Y., Huang, H. L., \& Chen, S. L. (2011). Development of a laser-based measurement system for evaluation of the scraping workpiece quality. Optics and Lasers in Engineering, 49(8), 1045-1053. 